\title{
La responsabilidad social universitaria, el reto de su construcción permanente
}

\author{
José Beltrán-Llevador, Enrique Î́ñigo-Bajo y Alejandrina Mata-Segreda
}

\section{RESUMEN}

Mediante el presente diálogo compartido entre los autores, se analiza el concepto de responsabilidad y vinculación social de la universidad, dándole una reinterpretación a la luz de diversas posiciones conceptuales y políticas que van desde perspectivas de pretensión hegemónica globalizada hasta las más diversas expresiones sociales que demandan nuevas respuestas a esta institución. En síntesis, se enfatiza su propia naturaleza, cultivada a lo largo de ocho siglos, de cara a la necesidad de ejercer un liderazgo que promueva una mayor equidad en procura de la cohesión social como principio ético y razón social de la universidad.

Palabras clave: responsabilidad social universitaria, equidad, cohesión social.

José Beltrán-Llevador

jose.beltran@uv.es

Español. Doctor en Filosofía por la Universidad de Valencia. Director del Departamento de Sociología y Antropología Social, Facultat de Ciències Socials de la Universidad de Valencia. Temas de investigación: sociología de la educación, formación del profesorado, educación a lo largo de la vida y políticas educativas.

Enrique Íñigo-Bajo

inigo@cepes.uh.cu

Cubano. Doctor en Ciencias de la Educación por la Universidad de La Habana. Jefe del grupo Educación y Sociedad en el Centro de Estudios para el perfeccionamiento de la Educación Superior de la Universidad de la Habana. Temas de investigación: calidad de los graduados de la educación superior (ES) en Cuba, tendencias del desarrollo universitario, educación comparada, evaluación y acreditación universitaria, gestión universitaria (procesos y calidad), acceso y permanencia en la ES. de la Facultad de Educación de la Universidad de Costa Rica. Temas de investigación: políticas educativas, formación de profesionales en Educación, orientación vocacional y educación ambiental. 


\section{A responsabilidade sociall universitária, o desafio da sua construção permanente}

\section{RESUMO}

Através do presente diálogo compartilhado entre os autores, analisa-se o conceito de responsabilidade e vinculação social da universidade. Uma reinterpretação é abonada ao conceito à luz de várias posições conceituais e políticas, que incluem desde perspectivas de pretensão hegemónica globalizada, até variadas expressões sociais demandando novas respostas a essa instituição. Em síntese, a própria natureza, é enfatizada, cultivada ao longo de oito séculos, de face à necessidade de exercer uma liderança que promova maior equidade na procura da coesão social como princípio ético e razão social da universidade.

Palavras chave: responsabilidade social universitária, equidade, coesão social.

Social responsability of universities, the challenge of its continuos construction

\section{ABSTRACT}

This dialogue between the authors analyzes the concept of social responsibility and outreach of the university, reinterpreting it in light of different conceptual and political stances that span perspectives from globalized hegemonic ambitions to the most diverse social expressions and demand new answers from this institution. In short, it emphasizes its own nature, cultivated over eight centuries, facing the need to practice leadership that promotes greater equity and thus social cohesion as ethical principle and raison d'etre of the university.

Key words: social responsibility of universities, equity, social cohesion.

Recepción: 01/07/13. Aprobación: 07/05/14. 


\section{Introducción}

Una presentación sobre la noción de pertinencia social en relación con la responsabilidad y vinculación social universitaria ha de tener en cuenta que las ideas autóctonas se enmarcan en contextos más amplios, de los que reciben influencia y a los que también pueden influir, en un proceso en el que se entrecruzan las escalas locales y globales. En lo que sigue, pues, presentaremos una breve contextualización de la cuestión que nos ocupa, elaborada a partir de un diálogo compartido entre los autores, con el propósito de aportar a la reflexión que enriquezca el ejercicio de la función que la sociedad le ha asignado a la institución universitaria en lo referente a su compromiso social, de manera más renovada en las últimas décadas. Para ello se argumenta alrededor de dos aproximaciones sobre el tema, la primera que busca precisamente reinterpretar la visión renovada de la responsabilidad social universitaria, la segunda referida al ineludible acuerdo ético que procura vigencia a la universidad dentro de una sociedad.

La cuestión acerca de la pertinencia social de la educación superior, siendo relativamente reciente en la agenda internacional, tiene algunos precedentes interesantes. Así, en su ensayo Misión de la Universidad, Ortega y Gasset (1930) destacó la introducción de nuevas ideas y la promoción del espíritu crítico basado en el conocimiento, como dos de las funciones que la universidad debe asumir para participar en el proceso de modernización de las sociedades avanzadas. Las universidades, al desarrollar sus misiones de educación, investigación, y dimensión social, son actores claves para el nuevo modelo de sociedad basada en el conocimiento. Por su parte, el sociólogo José Medina Echavarría, cuya importante labor estuvo vinculada al Instituto Latinoamericano de Planificación Económica y Social (ILPES) en los años sesenta, ya advertía en Filosofia, educación y desarrollo que:

la educación puede ser un factor de desarrollo económico, pero no por eso constituirse en servidor exclusivo de esa tarea. Es decir, en los problemas que plantea [...] la relación entre educación, economía y sociedad, ésta conserva y debe conservar su autonomía tradicional, o sea el cumplimiento de las tareas específicas para las que nació y que mantuvo en todo tipo de sociedades, muy en particular en aquellas que en lenta decantación constituyeron la paideia de nuestra civilización occidental (Medina Echavarría, 1967: 116).

Una revisión a la literatura internacional sobre pertinencia social universitaria permite constatar una atención creciente en su relación con la responsabilidad y la vinculación social. Dentro de este panorama, y aludiendo a la influencia de corrientes internacionales, Llomovatte et al. (2006) ponen el foco de atención en las tendencias hegemónicas hacia la mercantilización del conocimiento, a partir del modelo de la "triple hélice", a saber, universidad, empresa, Estado, un triángulo que Medina Echavarría ya había anticipado, con una perspectiva antagónica, al hablar de educación, economía y sociedad. Este concepto, propuesto por Loet Leydesdirff y Henry Etzkowitz, cristalizó a partir de la Primera Conferencia sobre la Triple Hélice realizada en Amsterdam en enero de 1996, y Latinoamérica no permaneció ajena a su influencia (http://www. leydesdorff.net/th2/).

Como sucede con otros conceptos presentes en los discursos referidos al uso de la educación, el concepto de pertinencia social es objeto de controversia. Algunos autores critican el uso espurio de "pertinencia”, aplicado a la educación superior, cuando va asociado a una concepción centrada exclusivamente en el desarrollo económico o en los requerimientos de demandas externas. Naidorf, por ejemplo, nos recuerda que la noción de pertinencia de educación superior comienza a ser introducida:

por los documentos especializados de la UNESCO desde 1995, y es reforzada sistemáticamente a partir 
de 1998, en ocasión del Encuentro mundial sobre la enseñanza superior en el siglo XXI. Allí la pertinencia social de la universidad es definida como la capacidad de responder a las necesidades o problemas sociales. Esta visión se enmarca en la propuesta de las nuevas formas de producción de conocimiento cuya característica central reviste en que la definición del problema de investigación debe surgir a partir del contexto de aplicación [...]. El conocimiento pertinente surge a partir de las demandas y se enmarca en las miradas que enfocan su interés en lo regional y lo local. La función instrumental, desde esta perspectiva, se vuelve prioritaria (Naidorf, 2011: 48).

Efectivamente, como señalan Ramalho y Beltrán (2012: 39), entre 1994 y 1995 el Banco Mundial (Higher Education: the Lessson of Experience) y la UNESCO (Policy for Change and Development in Higher Education) coincidían en la importancia de las relaciones entre educación superior y sociedad, con conclusiones opuestas. La perspectiva de la UNESCO sostenía que la educación superior es un bien público y que es necesaria una internacionalización desde el principio de solidaridad y cooperación. Las prioridades del Banco Mundial eran otras, orientadas a una mayor integración con el sector privado y empresarial, que deberían participar en los órganos de gobierno de las universidades, y a una mayor atención al mercado, potenciando la diversificación institucional, con la privatización como prioridad. Estas dos perspectivas resumen tendencias y tensiones que continúan en la actualidad y que influyen en la resignificación del rol de la educación superior en la sociedad.

La dificultad del término estriba, en buena parte, en su carácter multidimensional y relativo, tal y como sucede con el concepto de "calidad", por ejemplo. Y eso hace que los conceptos sean más fácilmente instrumentalizados en su uso para sostener incluso posiciones antagónicas. Para evitar cualquier tipo de equívocos, sugerimos como punto de partida el siguiente significado de pertinencia social de la educación superior, siguiendo a De Ketele (2008: 55): "Para una institución de educación superior, la pertinencia supone inscribir sus objetivos dentro de un proyecto de sociedad". Ahora bien, como este autor advierte, una primera concepción consistiría en decir que la educación superior produce y aplica el conocimiento para favorecer el desarrollo de la sociedad, entendiendo que el desarrollo científico y tecnológico crea una sociedad del conocimiento. En realidad la traducción de esta idea, excesivamente simple y amplia en su formulación, puede acabar oficializándose (por parte de organismos macroeconómicos) en doctrina que se puede resumir así: puesto que el desarrollo económico de las regiones depende de los esfuerzos invertidos por las instituciones que producen y difunden nuevos conocimientos científico-tecnológicos, entonces tendrían que orientarse al mundo de los negocios, que crea más riqueza cuanto más consigue explotar esos conocimientos. Esta concepción, sin embargo, se ve desmentida por una serie de constantes que confirman que la riqueza que produce la empresa sobre la base de la transferencia de nuevos conocimientos científicos y tecnológicos sirve de forma exclusiva a los intereses de los más ricos y de los más poderosos en detrimento de los más débiles e incluso del futuro de la humanidad.

Pretender que el ser humano no genere controversia o que el significado de sus palabras sea uno y nada más, es tarea inútil y contraria a la libertad de pensamiento que promueve la misma universidad. Pero en abono a nuestra posición con respecto a la pertinencia universitaria por medio de su responsabilidad y vinculación social, traemos a este texto las palabras del educador Isaac Felipe Azofeifa cuando reflexiona sobre el futuro de la universidad en el siglo XXI. Para él la función fundamental de la universidad "[...] es compleja y una sola a la vez: generar pensamiento original junto con el debate consiguiente y mantenerse en la vanguardia del pensamiento nacional en busca permanente de la libertad, para lograr la triple conquista de identidad, autenticidad 
y soberanía cultural de la nación" (Azofeifa, 1994: 37). Esta visión nos trae a la memoria el paradigma del poder transformador de la universidad sobre la sociedad y es a partir de lo que le es propio, a saber, la generación de conocimiento, que puede impulsar dicha transformación como obligación ética: se trata de llevar el conocimiento a todo el que lo requiera.

Hecho este preámbulo, nos enfocaremos ahora en tratar de comprender mejor lo que es responsabilidad social universitaria y vinculación social como conceptos propios de la pertinencia de la universidad.

\section{El debate sobre la responsabilidad social universitaria}

La universidad siempre ha desarrollado su quehacer a partir de una conciencia social, en algunos momentos orientada hacia la producción de conocimiento exclusivamente, por lo que fue calificada como "torre de marfil", pero a pesar de eso construyendo conocimiento para la humanidad, avanzando en el tiempo con visiones relacionadas con la formación de los profesionales que requiere la sociedad, la respuesta a las necesidades sociales mediante la investigación y la extensión, la incidencia política, la transferencia tecnológica, el desarrollo humano sostenible, entre otros conceptos que permanentemente ponen al día las formas en que la universidad ejerce su responsabilidad social. En definitiva, esa vinculación social de la universidad, como principio de responsabilidad, ha existido secularmente aunque a partir de diferentes énfasis.

En la actualidad presenciamos el resurgimiento del debate acerca de la responsabilidad social de la institución universitaria. La educación superior, en tanto que bien público, es responsabilidad de todas las partes interesadas, en particular de los gobiernos, tanto en su control cuando de iniciativa privada se trata, como en su financiamiento. En contraste, ante la complejidad de los desafíos mundiales, presentes y futuros, la educación superior tiene la responsabilidad social de hacer avanzar la comprensión de problemas con dimensiones sociales, económicas, científicas y culturales, así como la capacidad de hacerles frente (UNESCO Conferencia Mundial de la Educación Superior, 2009). La educación superior debe asumir el liderazgo social en materia de creación de conocimientos de alcance mundial para abordar retos asociados a la desigualdad, a la explotación del planeta y a la comprensión humana, entre otras situaciones de urgente atención.

En este contexto el compromiso de la universidad exige de su habilidad y efectividad para responder a las necesidades de transformación de la sociedad donde está inmersa, mediante el ejercicio de sus funciones básicas: docencia, investigación y extensión/proyección social. Estas funciones deben estar respaldadas por la búsqueda de la promoción de la justicia, la solidaridad y la equidad social, mediante la construcción de respuestas exitosas para atender los retos que implica promover el desarrollo humano integral (Guillén, 2012).

De esta corriente naciente con el siglo XXI, y en concordancia con el principio de responsabilidad que ha caracterizado a la institución universitaria desde su creación hace más de ocho siglos, vale la pena rescatar algunas ideas que nos permitan, una vez más, reflexionar sobre las nuevas expresiones sociales que demandan nuevas respuestas a la universidad. Cabe destacar la importancia de no permitir que la institución universitaria sea concebida como una empresa que ofrece un tipo particular de mercancía (mercancía compleja en el lenguaje de la economía neoliberal), sino manteniendo como suyas las mismas condiciones de identidad, autenticidad y soberanía cultural que ha buscado promover en la sociedad.

En la actualidad, algunos autores introducen el concepto de responsabilidad social universitaria (RSU) como una nueva condición de esta institución, comparándolo con el concepto de responsabilidad social empresarial que se añade mucho más recientemente. Un paso más allá, De la Cuesta y Sánchez Paunero 
(2012) introducen el concepto de responsabilidad social universitaria 2.0, en relación con el desarrollo de la Web 2.0 y de los últimos avances en tecnologías de la comunicación. Influidos de igual manera por la teoría del desarrollo social empresarial, otros autores ponen el acento en la necesidad de mejora social. Así, Martínez de Carrasquero, Mavárez, Rojas y Carvallo (2008: 4), de la Universidad de Zulia, sostienen que para estar a tono con los nuevos tiempos,

la universidad requiere definir la visión integral de sus funciones académicas: docencia, investigación y extensión sobre un nuevo paradigma cuyo uno de sus mejores soportes es una comunicación oportuna como eje transformador que le permita, a través de la creación y difusión del conocimiento lograr la correspondencia entre lo que la sociedad demanda por una parte y la coherencia interna que debe reinar en la universidad, por la otra, en la búsqueda de la pertinencia social que aspira el contexto de las instituciones de educación superior, tratando de hacer compatible el discurso con la acción.

Agregan estos autores que la RSU es una estrategia de gestión que trata de mantener un enfoque holístico sobre la propia organización universitaria, y concibiendo iniciativas interdisciplinarias (sinergia entre facultades y dependencias universitarias) e interinstitucionales (asociación de varias funciones de la estructura institucional). El papel de la universidad no es el de sustituir al Estado o a las Organizaciones no Gubernamentales (ONG) de desarrollo, sino el de asumir cada vez mejor su rol de formación superior integral con fines éticos y en pro del desarrollo sostenible de su comunidad, precisando no concebir las relaciones entre la universidad y el entorno social en términos de donación o ayuda unilateral.

La responsabilidad social universitaria se hace realidad cuando la organización toma conciencia de sí misma, de su entorno y de su papel en dicho entorno; presupone la superación de un enfoque egocéntrico (Ayala García, 2011). Es esta idea, precisamente, la que recoge la argentina Silvia Gabriela Vázquez (2011) al señalar que la responsabilidad social universitaria responde al "¿qué puedo hacer yo?" convirtiendo el yo en nosotros y las utopías en acciones.

De lo anterior se infieren dos componentes complementarios asociados al tema de la pertinencia universitaria. Se trataría no sólo de promover la mejora social como fue expuesto más arriba, sino también de la importancia de que la universidad se sienta y sea parte de esa misma sociedad. Se trata de tener abierta la ventana para que la universidad pueda mirar hacia afuera pero a la vez que pueda ser mirada desde afuera como todo buen vecino que es parte de su comunidad. Esta metáfora, utilizada a lo largo de la reflexión desarrollada en el encuentro interuniversitario "Universidad: equidad y cohesión social" celebrado en 2012 en la Universidad de Costa Rica, ubica a la institución no como centro, no como eje, no desde afuera sino como parte del entramado social sobre el cual es tan responsable como los otros actores sociales, respetando cada uno de los espacios que éstos ocupan, sin sustituirse unos a otros (mucho menos pretender que la universidad sustituya al Estado), participando y promoviendo a la vez, la construcción de un proyecto social consensuado.

En este permanente construir del concepto de pertinencia en general y de pertinencia de la educación superior, surgen nociones asociadas que enriquecen, complementan y materializan algunas de las acepciones de la pertinencia entendida como responsabilidad social universitaria. De acuerdo con Herrera (2008: 176), "el nuevo perfil de la RSU, que trasciende lo que comúnmente se ha denominado pertinencia, se refleja en el diseño de políticas educativas que tienden a estimular una mayor correspondencia entre el entorno y los objetivos fundamentales de las universidades". Y agrega Gaete que este tipo de responsabilidad se materializa a partir de tres perspectivas, a saber, gerencial o directiva, 
transformacional y normativa, interesándonos más en este contexto la transformacional, la que define el autor como "la tarea de liderar los aportes y reflexiones que permitan alcanzar una mejor sociedad, más equitativa y más justa con especial atención en un desarrollo más sustentable, en donde la universidad sea la institución capaz de mostrar cuáles son las mejores formas de construir una mejor sociedad" (Gaete, 2011: 116-117).

\section{La reinterpretación de la responsabilidad social universitaria}

Desde una perspectiva renovada sobre las tres misiones universitarias (docencia, investigación y extensión), y siguiendo la línea de pensamiento de Gaete (2011), la concreción de la responsabilidad social de esta institución es posible de lograr por medio de diferentes iniciativas en cuatro ámbitos: formación, investigación, liderazgo social y compromiso social. En el ámbito de la formación, destaca el aprendizaje-servicio que favorece la preparación de los estudiantes para su inserción en la sociedad como ciudadanos responsables, más allá de la simple adquisición de competencias profesional para su desempeño laboral. Con respecto a la investigación apela a lo acordado por la Cumbre Mundial sobre la Educación Superior de la UNESCO realizada en 2009, en la que se define la RSU como el medio que permite que la sociedad comprenda y pueda abordar mejor los diferentes problemas que la afectan y que tienen repercusiones en dimensiones sociales, económicas, científicas y culturales, destacando que las instituciones de educación superior deben asumir un liderazgo social en la creación de conocimiento estimulando el pensamiento crítico y la ciudadanía activa. Sobre el liderazgo social de la universidad, Gaete acude a la óptica crítica de Chomsky en su discusión sobre el papel de la responsabilidad social universitaria considerando su "función liberadora y subversiva que permita a la sociedad discriminar si las actuales instituciones de orden, autoridad y dominio son útiles en términos de los derechos y necesidades humanas fundamentales" (Chomsky, 2002: 116 en Gaete, 2011). El compromiso social lo concreta en la creación de estructuras solidarias y en políticas de cooperación universitaria al desarrollo, que van ganando una presencia creciente en educación superior tanto en los órganos de gestión como en los planes de estudios y en la atención a la educación para el desarrollo.

Este cambio de paradigmas, puede decirse que acelerado, tiene consecuencias importantes para la educación superior y su necesaria redefinición. Así, en la orientación de la universidad hacia el desarrollo y la cooperación, va adquiriendo cada vez más fuerza la articulación de nuevas formas de vinculación con la sociedad civil. Las instituciones de educación superior pueden explorar y ensayar estos nuevos caminos a partir de la reinterpretación de sus tres misiones, que ahora se integran en marcos más amplios: la docencia se integra en el marco de la formación; la investigación se integra en el marco del conocimiento, y la extensión se integra en el marco del servicio. Aún más, el cambio de paradigma no sólo es un factor determinante del progreso individual y social, sino que además confiere a la producción y difusión del conocimiento una función clave a la hora de configurar la posición de los países en el orden internacional. Desde la perspectiva de la comunidad universitaria, estas tendencias suponen la asunción de una responsabilidad cada vez mayor en materia de formación, investigación, estudio y servicios de orientación, transferencia de tecnología y educación permanente (Ramalho y Beltrán, 2012; Neave, 1998).

En cuanto a la formación, la universidad debe tener presente que para lograr un desarrollo social eficaz y sostenido, es de suma importancia que la labor universitaria se desarrolle sobre la base de una planeación y gestión estratégica de proyectos que partan de un diagnóstico del contexto social y de las necesidades prioritarias de la sociedad sobre 
la que se trabaja, que se diseñen y ejecuten con la metodología y acompañamientos académicos adecuados, y que se evalúen con indicadores pertinentes que permitan conocer tanto el impacto que esos proyectos tienen en la formación personal y profesional de quienes participan, como su impacto social e institucional. Además, las instituciones de educación superior comienzan a rediseñarse para ofrecer una formación continua, a lo largo de la vida. De este modo, pueden contribuir a la formación de la ciudadanía con formatos, modalidades y enfoques muy variados. Pero esta formación no ha de ser necesariamente unidireccional, sino que la propia universidad puede aprender y formarse a partir del diálogo y de la alianza con los actores de la sociedad civil: esta relación puede enriquecer a las propias instituciones educativas al tiempo que contribuye al desarrollo humano y social contando para ello con los propios sujetos sociales (Navarro, 2011; Ramalho y Beltrán, 2012).

Desde el punto de vista del conocimiento, las instituciones de educación superior pueden promover proyectos de investigación conjuntos con actores de la sociedad civil (sindicatos, cooperativas, ONG, movimientos sociales) que tengan en cuenta sus necesidades y puntos de vista. Un ejemplo de este intento de convergencia entre universidad y sociedad civil lo ofrece la red Living Knowledge (www.livingknowledge.org) a través del movimiento de las "tiendas científicas" que favorece el acercamiento de los miembros de instituciones de educación superior a los problemas de la comunidad. Otro ejemplo es la participación de la educación superior para producir y movilizar conocimiento colaborando directamente para procurar que se alcancen los objetivos de desarrollo del milenio (Ramalho y Beltrán, 2012). Ya había planteado García-Guadilla (2002) desde hace una década que la principal característica del nuevo contexto en que la universidad produce conocimiento es el énfasis que se pone en quienes producen conocimiento y quienes se apropian del conocimiento. Las personas usuarias del conocimiento generado por la universidad no sólo son sus estudiantes sino las comunidades y los otros niveles del sistema educativo.

Planteamos que la dimensión social de la universidad, que tradicionalmente ha encontrado su expresión explícita, principalmente, en los espacios y las actividades que desarrolla en su entorno, debe estar imbuida por una actitud de servicio. Es necesario un estrecho acercamiento entre las comunidades y las actividades de acción social, proyección social o extensión universitaria, o como se denominen aquellas actividades que realiza la universidad fuera del espacio académico. Deben constituirse en espacios para la difusión del conocimiento y el intercambio de experiencias como actividades de servicio tendientes a procurar el bienestar general de la comunidad y la satisfacción de sus necesidades. Dentro del marco del servicio, cada vez son más frecuentes las prácticas de los estudiantes implicándose en una comunidad local. Hasta ahora el acento se venía poniendo en la formación de los estudiantes más que en el servicio que podían prestar a la comunidad. Sin embargo, ahora la perspectiva tiende a cambiar la manera en que la formación del estudiantado se lleva a cabo a través de experiencias reales y de servicios realmente prestados a la comunidad, y no de servicios recibidos de la comunidad (Aponte, 2009; Ramalho y Beltrán, 2012). La noción de aprendizaje-servicio refleja este nuevo enfoque de la función de servicio, cada vez más extendida y con muchos ejemplos de buenas prácticas, y supone una forma alternativa de vinculación con la sociedad civil. Este es uno de los enfoques asumidos, entre otros, por la red Universidades Construye País, de Chile (Villar, 2010). Es además la práctica propia del Trabajo Comunal Universitario que se desarrolla en la Universidad de Costa Rica, el cual busca despertar la conciencia social del estudiantado, ayudar a las comunidades a identificar sus problemas y juntos desarrollar soluciones y sensibilizarle para que fortalezcan procesos de retribución hacia las comunidades (http:// 
accionsocial.ucr.ac.cr/web/tcu/trabajo-comunall).

La Declaración del Congreso Internacional de Rectores Latinoamericanos y Caribeños, celebrada en 2007 en Belo Horizonte, Brasil (UNESCO-IESALC, 2007), consideró que el camino de reflexión, profundización y difusión de la responsabilidad social debe ser capaz de atender los desafios desde la propia identidad de las universidades; esta declaración igualmente afirmó que la universidad en América Latina está llamada a reforzar sus funciones de servicio a la sociedad y de un modo más concreto hacia la erradicación de la pobreza, la intolerancia, la violencia, el analfabetismo, el hambre, el deterioro del medio ambiente y las enfermedades, es decir, a responder a los desafios de alineación y colaboración con los objetivos del milenio y de la educación para todos.

En una reunión de trabajo realizada en marzo de 2008 en Medellín, que prolongaba las reflexiones del Congreso de Belo Horizonte (Villar, 2010: 28-29), se llegó a la conclusión de que el concepto de responsabilidad social universitaria debe ser construido por cada universidad a partir de un proceso participativo de todos los actores externos e internos pero teniendo en cuenta una serie de grandes condiciones o directrices acordadas entre quienes participaron.

Respecto a su finalidad se consideró que la responsabilidad social universitaria:

- Orienta a las universidades hacia una clara conciencia de su misión: una opción ético política de contribución al desarrollo humano y sustentable, la equidad, la inclusión social, los derechos humanos y la cultura de la paz.

- Invita y posibilita generar políticas y estrategias adecuadas que aseguren la congruencia de sus procesos de docencia, investigación, extensión y gestión adecuadas con dicha misión y a la cual direccione su capital humano, relacional, intelectual, tecnológico y económico.

- Propicia una adecuada concepción de la autonomía enfatizando la capacidad de tomar sus propias decisiones responsabilizándose de sus procesos e impactos y a la vez atendiendo a los requerimientos, interpelaciones y necesidades de todos.

- Crea condiciones para una cultura de la observación y escucha, propia y externa, que genere el diagnóstico, la evaluación de sus procesos, acciones e impactos presentes y esperados, tanto cognitivos como educacionales, sociales $\mathrm{y}$ medioambientales.

- Genera la transparencia y la rendición de cuentas tanto a la comunidad universitaria y la sociedad en general a la que se debe.

- Permite la integración curricular de la RS en forma de modelo educativo, programas específicos de ética aplicada y RS, y procesos evaluativos propios o característicos así como de técnicas y metodologías pedagógicas ad hoc (Villar, 2010: 29).

En un escenario de sociedades crecientemente complejas y de elevada reflexividad sociológica, vale la pena subrayar otro elemento que destaca la importancia de la tercera misión universitaria en relación con su responsabilidad y vinculación social. La tercera misión, identificada bien como servicio en general o como extensión (atendiendo a su concreción en departamentos o instancias de extensión universitaria) puede considerarse una misión trascendental - no confundir con "trascendente" - en el sentido estrictamente filosófico del término, en la acepción que Kant le otorgó al término en su Crítica de la razón pura. Podría decirse que constituye una clase de conciencia social institucional, en este caso, de carácter universitario. A través de la extensión universitaria, la universidad puede determinar el origen, la extensión y la validez objetiva del conocimiento y de la producción de conocimiento, es decir, puede pensarse a sí misma, desde la experiencia social, pero no más allá de los límites de la experiencia. O lo que es lo mismo, a través de la extensión universitaria, la universidad 
puede convertirse en una institución que, en la tarea de transferir y aplicar conocimiento a la sociedad, vincularse y comprometerse socialmente, aprende de la misma sociedad a la que sirve y a la que aplica su saber. No otra es la idea de la universidad como comunidad de aprendizaje; la educación es educarse, afirmó el fundador de la escuela hermenéutica H. G. Gadamer, una idea que, para el caso, podría traducirse en términos de que la universidad, al educar, se educa, aprende de la sociedad a la que educa y a la que transfiere su conocimiento. De este modo, la responsabilidad social universitaria no consiste sólo en actuar sobre la sociedad, sino sobre la manera de comprender la sociedad. Una universidad transformadora y emancipadora tiene que saber transformarse a sí misma, tomando conciencia a su manera de su visión de la sociedad. Esta búsqueda permanente de transformación interna para permitirle a la universidad dar respuesta a las exigencias sociales le exige a la institución potenciar su capacidad de aprendizaje mediante la formación de una comunidad de aprendizaje asociativo que le permita buscar, desarrollar y difundir nuevas prácticas en sintonía con estos nuevos escenarios sociales (Cohen, 2007).

Ahora bien, el espacio universitario interno está conformado, naturalmente, de lugares estratégicos en los que la responsabilidad social se hace efectiva. Vallaeys (2007) propone como el primero de ellos el constituido por la ciencia. Es en la universidad donde convergen la producción del saber científico (la investigación), la reproducción de este saber (trasmisión de conocimientos y formación de ciudadanos y ciudadanas profesionales que socializan las ciencias y tecnologías) y la información del gran público sobre el quehacer científico (universidad como espacio social abierto al debate). La actividad científica es una actividad social como cualquiera, ni neutral ni inocente que afecta a la ciudadanía global en su totalidad y que requiere del pluralismo y la racionalidad de la academia como garantía de transparencia y libertad.

Un segundo lugar identificado por este mismo autor, que apela a ser integrante consustancial de la vida universitaria como medio para el cumplimiento de la responsabilidad social, es la formación para la ciudadanía democrática. Constituye un espacio privilegiado por fomentarse y enriquecerse la capacidad de reflexión y decisión juiciosa en una sociedad en la que los medios de comunicación han demostrado su incompetencia para hacerlo (falta de voluntad, falta de independencia de criterio, falta de conocimiento). Esta tarea específica le significa a la universidad abrirse a la formación de personas más allá de las que formalmente ocupan sus aulas mediante la educación continua, la extensión docente, la facilitación de espacios de debate y reflexión ciudadana y la difusión de la cultura en todas sus dimensiones. Pero desde la perspectiva de la docencia se ha hecho evidente que son escasos los esfuerzos por hacer explícita la formación ciudadana de los futuros y las futuras profesionales a pesar de que el ejercicio ciudadano es inherente al ejercicio profesional. ¿Se desea evadir el riesgo de hacer caer a la universidad en activismo político y adoctrinamiento de estudiantes y otras personas miembros de la comunidad universitaria? Responsablemente la universidad debe orientar su estrategia de formación ciudadana a partir de la implementación del espacio público del debate en su propia comunidad dando cabida al ejercicio democrático informado en los términos en que su propia constitución lo establezca. Lo único que debe temerse en este caso es promover una universidad que asuma una posición "positivista-cientificista" que, negando ser una posición, desde la altura de la pretendida neutralidad axiológica impida cualquier crítica contra ella y contra la ciencia y redunde en el desarrollo de una actividad científica sin fiscalización ética.

Como tercer nicho para la concreción de la responsabilidad social universitaria Vallaeys (2007) propone la formación para el desarrollo. Su quehacer se centra en orientar la formación general y especializada de cada estudiante que promueva el desarrollo 
justo y sostenible. El autor define este nuevo perfil profesional a partir del estímulo al compromiso y la solidaridad como factor primordial para el futuro ejercicio laboral; del estímulo al conocimiento y la información necesaria para contextualizar el saber especializado; del desarrollo de la capacidad de escucha, de intercambio y empatía en el marco de la ética del diálogo y de la competencia necesaria para saber ser ciudadano, es decir, saber gobernar y ser gobernado.

Los anteriores tres elementos, ciencia, ciudadanía y desarrollo, articulan la mirada hacia adentro de la universidad con miras a dar una respuesta socialmente responsable, mirada que le exige a la institución modificar las formas de gestionarse para contribuir a la formación profesional, como se ha hecho evidente. Adicionalmente esta formación se verá impactada por la calidad de la vida universitaria que se promueva para cada uno y cada una de sus miembros (¿ciudadanos?). La calidad de vida que se genere en el claustro universitario desde la perspectiva de responsabilidad social será elemento determinante para la asimilación del conocimiento y sus implicaciones éticas por parte del estudiantado, además que contribuirá al proceso de socialización que innegablemente aporta la universidad.

\section{La responsabilidad social universitaria vista desde una perspectiva ética}

Hemos de enmarcar este tema dentro de la idea que plantea Kliksberg (2004) sobre el tema de la "sed ética" como exigencia del mundo actual. Ninguna institución social puede abstraerse del escrutinio de la ciudadanía. Las facilidades actuales para el acceso a la información han dado una nueva configuración a la participación ciudadana la cual ha sobrepasado los medios tradicionales de hacerse efectiva, como el acto de elegir a sus representantes, para dar espacio a una especie de auditorías ciudadanas más activas e inmediatas. La universidad como institución social no está exenta de esta realidad por lo que su vinculación social debe darse en doble vía, escuchando las voces de las demandas sociales y mostrando a la vez a estas voces los productos de su actuación. En este sentido, tras el proceso de globalización, al igual que en otras instituciones sociales, han ido cobrando fuerza la conciencia ecológica, el fortalecimiento del derecho a la información y otros principios éticos compartidos mundialmente, la universidad se ha visto urgida de revisar las exigencias de los grupos de interés y su comportamiento social pues, como sostiene Momberg (2006), con el devenir del tiempo la visión de responsabilidad social de toda institución ha ido progresivamente incorporando temas nuevos a su contenido de manera que se redefinen sus prioridades y se asume una preocupación creciente por problemas cada vez más generales, en concordancia diríamos con esta nueva dinámica social.

Sin embargo, lo anterior no constituye la única razón por la que la institución universitaria deba dar cumplimiento a su compromiso ético. Ha propuesto Amartya Sen (2004) que la naturaleza de los valores de cada persona y de su colectividad, y sus impactos, dependerá siempre de las instituciones que les rodean, de ahí que el compromiso ético con el desarrollo depende básicamente de la institucionalidad, y añadimos que dentro de esta institucionalidad se encuentra la misma universidad. Debe entenderse entonces que al cumplir con sus obligaciones éticas de cara a las demandas sociales, la universidad se convierte en modelo que impacta en alguna medida la formación ciudadana.

La sed de ética de y hacia las instituciones se encarna hoy en lo que llamamos responsabilidad social como ética de la responsabilidad. Lo que antes llamábamos "participación y proyección social" de la universidad, a la luz actual se llamaría responsabilidad social universitaria. Como valor agregado del concepto está el empeño por superar la visión filantrópica para centrarse en una gestión de impactos, es decir, rendición de cuentas en cuanto a los impactos 
humanos, sociales y ambientales (Vallaeys, 2006). Podemos entender en este contexto que al trascender de la proyección social voluntaria a la RSU, se trasciende de una voluntad institucional de expresar valores de solidaridad a una condición constitutiva tanto en la formación humana y profesional que provee como en la construcción de conocimientos.

Si bien lo anterior en la actualidad forma parte del discurso cotidiano de la institución universitaria y ha venido ocupando un papel preponderante en programas de extensión universitaria y de servicio social, la reflexión desarrollada mediante Virtual Educa 2007-Brasil con respecto al caso de la Universidad Nacional Autónoma de México (UNAM) y probablemente con aplicabilidad a las otras universidades latinoamericanas, concluye que estas instituciones padecen graves problemas al no reflejar la presencia de sinergias entre la universidad, el sector gubernamental, el productivo y el social en general (Sánchez González, 2007). Producto de este foro de reflexión latinoamericana se define que aún hay tareas por desarrollar entre las cuales, desde la perspectiva ética, se destacan el mejoramiento de la calidad académica promoviendo la responsabilidad, los valores y el compromiso social; diseñar planes de estudio orientados hacia la formación de valores; fortalecer la identidad de las universidades como instituciones con firmes principios de responsabilidad social; consolidar foros y seminarios encaminados a analizar, desde la perspectiva ética, el impacto de las propuestas universitarias en la solución de los problemas que enfrenta la región y traducir la RS en la búsqueda del bienestar integral, de desarrollo humano sostenible y de sustentabilidad. Es decir, parte de las acciones encaminadas a favorecer la sinergia integradora de los diferentes actores sociales que se centran en acciones de corte ético como las mencionadas anteriormente.

Con la intención igualmente de brindar insumos que le permitan a la universidad vincularse socialmente desde una posición ética, Cohen (2007) propone que su responsabilidad social no parte de centralizar la problemática en un aspecto estrictamente técnico, económico o político sin tomar en consideración la dimensión ética. El rol universitario en el marco de la sociedad no puede omitir las implicaciones éticas de toda posición científica y técnica ya que pueden derivarse resultados regresivos para la comunidad tales como desigualdades e inequidad. La influencia universitaria debe favorecer la construcción de procesos sociales con identidad y sostenibilidad de manera que aseguren un verdadero empoderamiento desde la base social lo que indudablemente le exige a la institución ejercer su capacidad de valorar y tomar decisiones con miras a la mayor conveniencia de la colectividad. En suma, la ciencia y la técnica deben pasar por el filtro del compromiso social y ético de la universidad en lo que a difusión del conocimiento y formación profesional se refiere. No se trata de limitar el acceso al conocimiento o su desarrollo, se trata de impulsar la mejor apropiación de dicho conocimiento para el bienestar social. Tal y como se ha planteado, la institución universitaria no es ni apolítica ni neutral, su compromiso social debe ser claramente orientado hacia los más altos valores que promuevan las transformaciones requeridas para el mejoramiento social con igualdad.

Hemos hecho referencia a aspectos éticos que configuran y potencian la responsabilidad social universitaria y la hacen asumir su compromiso constitutivo en cada uno de los espacios sociales en los que se desenvuelve. Ahora es preciso analizar un elemento más que ha generado diversas interpretaciones por lo que la idea tiene tanto defensores como detractores. Nos referimos a la evaluación y acreditación universitaria, condición surgida en diferentes nichos que van desde la imposición de ideologías economicistas que buscan únicamente el saber utilitario que le pueda proveer la universidad, hasta posiciones autónomas en que es la propia institución la que participa y favorece el surgimiento de modelos de acreditación a los cuales se somete como 
compromiso ético. En este último caso se resalta el Sistema Nacional de Acreditación de la Educación Superior (SINAES) de Costa Rica cuya constitución fue acordada por cuatro universidades públicas y cuatro privadas del país, modelo construido por consenso entre las instituciones pero otorgándole la misma autonomía que constitucionalmente se le asigna a las universidades del país.

De acuerdo con Gort (2012), la evaluación de carreras y de instituciones de educación superior tiene como propósitos:

- reconocer fortalezas y debilidades,

- conquistar nuevos logros y consolidar aciertos institucionales que podrían deteriorarse,

- adecuarse a los cambios vertiginosos y anticiparse a retos futuros,

- dar seguimiento más fino de las políticas de educación superior y

- rendir cuentas sobre el aseguramiento de la calidad de los servicios que la universidad ofrece.

La última función requiere de la imagen externa que constituye la acreditación por razones obvias de visibilidad, pero sobre todo, por los antecedentes evaluativos en América Latina que evidencian la detección de problemas pero no de sus causas y su descripción de forma general, con prevalencia del diagnóstico sobre la docencia. Fue común, y probablemente aún lo sea, que la evaluación de instituciones o carreras era realizada por grupos de sujetos activos en pequeñas comisiones subordinadas a las autoridades universitarias con escasa o nula participación de agentes externos. Agrava la situación la excesiva formalidad en los procedimientos de recolección de los datos con abuso del cuestionario (Iñigo, 2010).

La idea de responsabilidad y vinculación social encierra una exigencia a la universidad en coherencia con sus propias tesis y es la necesidad de que las propias instituciones de educación superior sean evaluadas externamente. No se trata de procesos estandarizados de rendición de cuentas que ponen exclusivamente la atención en productos y resultados más que en procesos sino más enmarcados en una cultura de la evaluación. La cultura de la evaluación es una condición de la democracia educativa y de la educación democrática que debería ser asumida como un imperativo institucional. Solo desde una auténtica evaluación institucional universitaria se estará en condiciones de redefinir funciones, misiones y visiones en busca de un mayor y mejor compromiso con la sociedad (Martínez, 2007; Bueno y Casini, 2007).

Desde este compromiso, es necesario repensar los fines de la educación superior para que las universidades fortalezcan su accionar en cuanto a responsabilidad social. Esta reflexión puede contribuir decisivamente a una propuesta teórica basada en principios de acción orientados por este compromiso con la sociedad. Principios que si bien no siempre se plasmarán en respuestas definitivas, al menos tendrán en cuenta una serie de preguntas pertinentes, como las que se enumeran a continuación en concordancia con la intención de este ensayo de promover la reflexión sobre el tema que nos ocupa: ¿Cuál es el horizonte de sentido hacia el que debe encaminarse la universidad del siglo XXI, esto es, qué necesitamos, qué esperamos, qué exigimos de la universidad del siglo XXI? ¿Qué transformaciones estamos observando en las universidades en relación con la pertinencia social? ¿Puede servir la noción de pertinencia social para legitimar un proceso de ajuste acrítico o de adaptación social a requerimientos sociales? Si es así, ¿corre la universidad el peligro de disminuir el espacio de autonomía que le ha venido caracterizando? ¿Es y debe ser la universidad un actor relevante para el bien común? ¿Tienen en cuenta las universidades las "voces" de la sociedad hacia las que dirige su acción? ¿Dialogan suficientemente las universidades con los actores sociales para elaborar el conocimiento científico?

Desde el punto de vista de la acción, ¿están 
reconociendo, evaluando, registrando y transfiriendo las universidades las buenas prácticas que están llevando a cabo? ¿Están asumiendo las universidades la necesidad de considerarse no sólo instituciones que enseñan, sino también instituciones que aprenden? ¿Están dando las universidades el paso de la transmisión de conocimiento (explicación, transferencia) al compromiso con el conocimiento (implicación social, pertinencia social en su acepción más positiva)? ¿Cómo concilian las universidades la lógica de la competición con la lógica de la cooperación?

Desde el punto de vista imperativo, desde aquello que podemos hacer, ipueden las universidades convertirse en actores relevantes para "salir del imaginario dominante" y contribuir a la construcción de alternativas sociales guiadas por principios solidarios de equidad y justicia social? ¿Pueden las universidades contribuir a repensar otros escenarios, desde la imaginación instituyente? ¿Pueden alentar las universidades un nuevo contrato social?

\section{Consideraciones finales}

La universidad es un actor relevante para construir y anticipar escenarios alternativos encaminados a lograr una mayor equidad y cohesión social. Sin embargo, para que la universidad sea realmente transformadora y no meramente adaptadora, tiene que estar dispuesta a transformarse a sí misma a la vez que tiene que ser ella misma en su función o misión de servicio a los demás. Esta misión es también una visión de la realidad social con aspiraciones universitarias, que busca conciliar la unidad de la humanidad con la diversidad de formas de vida, para una ciudadanía plena. Le corresponde a la institución universitaria hacer una lectura apropiada de su entorno para así definir las vías por medio de las cuales debe poner su conocimiento y capacidad de acción al servicio de las comunidades. Es decir, tal y como se ha venido planteando, el concepto de responsabilidad social universitaria está en construcción permanente en virtud de la interacción que se establece entre universidad y sociedad, con miras a promover la cohesión social como fin primordial. Formación, investigación, liderazgo social y compromiso son los elementos sustantivos que determinan el formato de esta relación para hacer efectiva su incidencia social. 


\section{Referencias}

Aponte, Claudia (2009), "Propuesta de indicadores de evaluación de la función de proyección social/ extensión universitaria/interacción en la educación superior", en <http://www.ascun.org.co/documentos/ extensionpropuestaproyeccionsocial.pdf $>$ [consulta: noviembre de 2012].

Ayala García, Mauricio Osvaldo (2011), Responsabilidad social universitaria, en <http://www.redicces.org.sv/jspui/ bitstream/10972/91/1/Responsabilidad\%20social\%20 universitaria.pdf $>$ [consulta: noviembre de 2012].

Azofeifa, Isaac Felipe (1994), "Reflexiones sobre el proyecto de universidad de Costa Rica para el siglo XXI", en A. Gurdián (comp.), Proyecto de universidad para el siglo XXI, San José, Costa Rica, UCR.

Bueno Campos, Eduardo y Fernando Casani Fernández de Navarrete (2007), "La tercera misión de la universidad. Enfoques e indicadores básicos para su evaluación”, en Economía Industrial, núm. 366, pp. 43-59.

Cohen, Daniel (2007), "Desafíos de la responsabilidad social universitaria", en Razón y palabra, núm. 55, año 12, febrero-marzo.

De Ketele, Jean-Marie (2008), "La pertinencia social de la educación superior", GUNI, pp. 55-59.

De la Cuesta, Marta y David Sánchez Paunero (2012), Responsabilidad social universitaria 2.0., La Coruña, Netbiblo.

Gaete Quezada, Ricardo (2011), "La responsabilidad social universitaria como desafío para la gestión estratégica de la educación superior: el caso de España", en Revista de Educación, núm. 355, pp. 109-133.

García-Guadilla, Carmen (2002), "Nuevas formas del cambio en los albores del tercer milenio", en Tensiones y transiciones. Educación superior latinoamericana en los albores del tercer milenio, Caracas, Nueva Sociedad/CENDES.

Gort, Amelia (2012), “La variable 'pertinencia' en la evaluación y acreditación de carreras universitarias en Cuba", en Encuentro Interuniversitario Universidad: equidady cohesión social, San José, Universidad de Costa Rica, 12 y 13 de julio.
Guillén, León (2012), “La responsabilidad social universitaria: un estudio de caso aplicado a la Universidad Autónoma del Caribe en Barranquilla, Colombia”, en Memorias VIII Congreso Internacional de Educación Superior Universidad 2012, La Habana, Cuba.

Herrera, Alma (2008), "Responsabilidad social universitaria", en GUNI, pp. 176-177.

Iñigo, Enrique (2010), "Espacio de la evaluación y acreditación en la gestión de la calidad de las IES en América Latina", en VII Congreso Internacional de Educación Superior Universidad 2010), La Habana, Cuba, IESALC/UNESCO, del 8 al 12 de febrero.

Kliksberg, Bernardo (2004), Más ética, más desarrollo, Buenos Aires, Temas Grupo Editorial.

Llomovatte, Silvia et al. (2006), La vinculación universidadempresa: miradas críticas desde la universidad pública, Buenos Aires, Miño y Dávila.

Martínez de Carrasquero, Cynthia (2007), "La responsabilidad social universitaria como estrategia para la vinculación con su entorno social", Brasil, Virtual Educa.

Martínez de Carrasquero, Cynthia, Ramón Mavárez, Ligibther A.Rojas y Belkys Carvallo (2008), "La responsabilidad social universitaria como estrategia de vinculación con su entorno social", en Revista de Filosofia Jurídica y Política, vol. 15, núm. 3, pp. 81-103.

Medina Echavarría, José (1967), Filosofia, educación y desarrollo, México, Siglo XXI.

Momberg, Manuel Reyno (2006), "Responsabilidad social empresarial como ventaja competitiva", Tesis de grado en opción al título de Máster de la Universidad de Santa María, Chile.

Naidorf, Judith (2011), "Criterios de relevancia y pertinencia de la investigación universitaria y su traducción en forma de prioridades", en Revista de la Asociación de Sociología de la Educación (RASE), vol. 4, núm. 1, pp. 48-58.

Navarro, Ivette (201 1, "La planeación y gestión estratégica de la responsabilidad y el compromiso social: un 
reto para las universidades latinoamericanas. Red Iberoamericana de Compromiso Social y Voluntariado Universitario", en Boletín IESALC Informa, núm. 214.

Neave, Guy (1998), "Autonomía, responsabilidad social y libertad académica" Asociación Internacional de Universidades (AIU), <http://vademecumjuridicum. blogspot.com/2012/10/libertad-de-catedra-culturageneral.html> [consulta: noviembre de 2012)].

Ortega y Gasset, José (1930), Misión de la universidad, Madrid, Revista de Occidente.

Ramalho, Betania y José Beltrán (2012), "Universidad y sociedad: la pertinencia de educación superior para una ciudadanía plena”, en Revista Lusófona de Educação, núm. 21, pp. 32-52.

Sánchez González, Claudia Patricia (2007), “La responsabilidad social universitaria (RSU) en el contexto del cambio de la educación superior", Brasil, Virtual Educa, <http://desarrollo. ᄀut. ᄀedu. $\neg$ co/ $\neg$ tolima/ $\neg$ hermesoft/ $\neg$ portal/ $\neg$ home_1/ $\neg$ rec/ $\neg$ arc_29586.pdf $>$ [consulta: noviembre de 2012].

Sen, Amartya (2004), "Reanalizando la relación entre ética y desarrollo", en <http://www.iadb.org/etica [consulta: noviembre de 2012].

UNESCO (2009), "La responsabilidad social de la educación superior", en Conferencia Mundial de la Educación Superior. París, 2009. Comunicado final, <http://www. unesco.org/education/WCHE2009/comunicado_ es.pdf> [consulta: noviembre de 2012].

UNESCO-IESALG (2007), Declaración del Congreso Internacional de RectoresLatinoamericanos y Caribeños, Belo
Horizonte, Brasil.

Vallaeys, François (2006), "Breve marco teórico de responsabilidad social universitaria", en Vallaeys y Carrizo, Responsabilidad Social Universitaria [CD interactivo], Red de Ética y Desarrollo, Banco Interamericano de Desarrollo.

Vallaeys, François (2007), Responsabilidad social universitaria. Propuesta para una definición madura y eficiente, México, Instituto Tecnológico de Monterrey.

Vázquez, Silvia Gabriela (2011), "Mitos y objetivos de la responsabilidad social universitaria", Boletin IESALC, núm. 214, pp. 1-6.

Villar, Javier (2010), Responsabilidad social universitaria: nuevos paradigmas para una educación liberadora y humanizadora de las personas y las sociedades, Chile, Universidad Católica de Temuco.

\section{Sitios web consultados}

Emergence of a Triple Helix of University-IndustryGovernment Relations, Science and Public Policy, núm. 23 (1996) 279-86, <www.leydesdorff.net/th2/> [consulta: noviembre de 2012].

Living knowledge <www.livingknowledge.org> [consulta: noviembre de 2012].

Universidad de Costa Rica, Trabajo comunal, <accionsocial.ucr.ac.cr/web/tcu/trabajo-comunal> [consulta: noviembre 2012].

\section{Cómo citar este artículo:}

Beltrán-Llavador, José, Enrique Iñigo-Bajo y Alejandrina Mata-Segreda (2014), "La responsabilidad social universitaria, el reto de su construcción permanente", en Revista Iberoamericana de Educación Superior (RIES), México, UNAM-IISUE/Universia, vol. V, Núm. 14, pp. 3-18, http://ries.universia.net/index.php/ries/article/view/367 [consulta: fecha de última consulta]. 